\title{
COMPUTATION OF CONTINUED FRACTIONS WITHOUT INPUT VALUES
}

\author{
P. SHIU
}

\begin{abstract}
An algorithm for the computation of the continued fraction expansions of numbers which are zeros of differentiable functions is given. The method is direct in the sense that it requires function evaluations at appropriate steps, rather than the value of the number as input in order to deliver the expansion. Statistical data on the first 10000 partial quotients for various real numbers are also given.
\end{abstract}

\section{INTRODUCTION}

There is a well-known simple algorithm for the development of a real number $\alpha$ into a continued fraction. Regardless of how $\alpha$ has been specified, its actual value is required as an input for the algorithm, which terminates if and only if $\alpha$ is rational. In practice, when the algorithm is applied to an irrational number $\alpha$, an input rational approximation for $\alpha$ has first to be computed. This input value is often given as a truncated digital approximation, on which exact rational arithmetic is then performed to deliver the continued fraction expansion. When there are many digits in the input value, the arithmetic involved in obtaining the expansion is rather tedious, especially in the initial stages of the algorithm. This is because exact integer-arithmetic has to be carried out on very large numbers at each stage in order to preserve the complete quotient, which is required for the calculation of the remaining partial quotients. In $1938 \mathrm{D}$. H. Lehmer [9] gave a modification of the algorithm by dividing the computations into a suitable number of sections, inside which the arithmetic involved is more manageable, so that the modified algorithm can be executed much more speedily. Nevertheless, the computation of a large number of partial quotients for a given real number $\alpha$ is still a difficult task, and for this and other reasons, A. J. van der Poorten and J. Shallit [14] have remarked that "It is notorious that it is generally damnably difficult to explicitly compute the continued fraction of a quantity presented in some other form."

It was proved by Lagrange that a necessary and sufficient condition for the sequence of partial quotients for $\alpha$ to be eventually periodic is that $\alpha$ is a quadratic irrational. Moreover, there is an efficient algorithm which, from the input of the three integer coefficients of the quadratic function defining $\alpha$, determines the period and delivers all the partial quotients in the period for

Received by the editor May 5, 1994 and, in revised form, August 2, 1994.

1991 Mathematics Subject Classification. Primary 11A55, 11K50.

Key words and phrases. Direct algorithm, partial quotients, Khintchine's constant. 
$\alpha$. Furthermore, once all the partial quotients have been found, it is easy to construct a subsequence of convergents which exhibits quadratic convergence to $\alpha$; see, for example, [16]. This method of obtaining the continued fraction expansion for $\alpha$ without an input value is much superior to the method based on the input value, especially since there is no a priori knowledge on how accurate the input approximation has to be in order to deliver all the partial quotients in a period. Indeed, the length of the period cannot, or at least should not, be determined in this indirect way.

Very little is known about the continued fraction expansion for $\alpha$ when it is algebraic with degree exceeding 2 , or when it is transcendental. In particular, it is not known if the sequence of partial quotients associated with $\alpha=\sqrt[3]{2}$, or $\alpha=\pi$, is bounded or not. Suppose that $\alpha$ has been defined as the zero of a differentiable function $f(t)$, for which the values at rational points $t$ can be computed with arbitrary accuracy. As we shall see, it is possible to compute the continued fraction expansion for $\alpha$ directly from $f(t)$, instead of having first to compute an approximation to $\alpha$ from $f(t)$ as an input. It will be convenient to call the original algorithm the basic method, Lehmer's modification the indirect method, and our new algorithm the direct method. The argument used in obtaining the direct method can be interpreted as an extension of that used by Lehmer, with the important difference that we have eliminated the need for an input value for $\alpha$. We shall give a rational-arithmetic algorithm which delivers the partial quotients for the continued fraction expansion for an algebraic number $\alpha$ of degree exceeding 2 . The method also works for a transcendental number that has been defined as the zero of a function for which the logarithmic derivative at a rational point can be computed with arbitrary precision.

A comparison in computing times being used shows that the direct method is superior in the following sense. Bearing in mind that function evaluations are required for the direct algorithm whereas external input values are readily supplied for the indirect algorithm, the computing times for a modest number of partial quotients using the two methods are similar, whereas it becomes prohibitively long for the basic algorithm. More specifically, when the algorithms have been implemented on a small machine, the computing time for 10000 partial quotients for an algebraic number is only a few minutes. The time taken for a transcendental number which is the zero of a function whose evaluation is not difficult, such as $\sin t$, which has a zero at $\pi$, is also only a few minutes. We shall say more on the advantage of such modifications on the basic method in $\S 4$, where we also mention the metric theory of continued fractions due to A. Khintchine, P. Lévy and others (see [5, 6, 11]). Results of computations for the partial quotients for various real numbers are given in the last section.

\section{REMARKS ON LEHMER'S METHOD}

The basic method for the continued fraction expansion of a real number is essentially the same as the Euclidean algorithm for the computation of the greatest common divisor of two integers. Lehmer [9] explained his indirect method as a modification of the Euclidean algorithm applied to two real numbers. In fact, his argument can be simplified and the method further enhanced by making use of an exact formula for the complete quotient. Let $\alpha$ be a real number, 
and define the sequence of partial quotients $\left(a_{n}\right)$ for $\alpha$ by the iterative scheme $\alpha_{0}=\alpha, \quad a_{0}=[\alpha], \quad \alpha_{n+1}=\frac{1}{\alpha_{n}-a_{n}}, \quad a_{n+1}=\left[\alpha_{n+1}\right], \quad n=0,1,2, \ldots$,

which terminates when $a_{n}=\alpha_{n}$ for some $n$, and this happens when and only when $\alpha$ is rational. This is the basic method for the delivery of the partial quotients, and, as an algorithm, the input value for $\alpha$ has to be a rational number, so that the algorithm will terminate. When this rational number has a large numerator and denominator, the arithmetic involved in the algorithm for $a_{n}$ will be lengthy, and Lehmer's idea is that the initial section of values for $a_{n}$ can still be obtained if we use as input an approximation with a smaller denominator, which will then speed up the calculations. Suppose now that $a_{n}$ has been computed for $n \leq N$ from an initial approximation to $\alpha$, and we need further values for $a_{n}$. This will, of course, require a more accurate input value for $\alpha$, and we need to apply it in such a way that the process can be continued by delivering the next section of partial quotients, namely those $a_{n}$ for $n>N$. This can be achieved by calculating also the convergents to $\alpha$, so that an updated version of the complete quotient can be calculated for the new input value for $\alpha$.

We use Perron's notation for continued fractions, and write

$$
\alpha=\left[a_{0}, a_{1}, \ldots, a_{n}, \ldots\right]=\left[a_{0}, a_{1}, \ldots, a_{n}, \alpha_{n+1}\right],
$$

where

$$
\alpha_{n+1}=\left[a_{n+1}, a_{n+2}, \ldots\right]
$$

is the $(n+1)$ st complete quotient for $\alpha$. When the values $a_{n}$ have been computed for $n \leq N$, we can use this formula for the complete quotient to find more partial quotients, provided we can have a good estimate for $\alpha_{N+1}$. This good estimate can be obtained from the revised input approximation to $\alpha$ together with its last convergent $x_{N} / y_{N}$, which will also need to be computed. The convergents $x_{n} / y_{n}$ are given by the iterative formula

$$
\begin{array}{lll}
x_{0}=a_{0}, & x_{1}=a_{0} a_{1}+1, & x_{n+1}=a_{n+1} x_{n}+x_{n-1}, \\
y_{0}=1, & y_{1}=a_{1}, & y_{n+1}=a_{n+1} y_{n}+y_{n-1},
\end{array}
$$

and the formula relating the complete quotient and the convergent is

$$
\left|\frac{x_{n}}{y_{n}}-\alpha\right|=\frac{1}{y_{n}\left(\alpha^{\prime} y_{n}+y_{n-1}\right)}, \quad \alpha^{\prime}=\alpha_{n+1},
$$

from which $\alpha^{\prime}$ can now be calculated from the new input value for $\alpha$. Lehmer did not mention this exact formula for $\alpha^{\prime}$, which would have given a simpler and better modification. For example, the analysis on exactly how many new partial quotients can be delivered from the updated value of the complete quotient can be much simplified, and we give this in the next section. The process can now be repeated in a systematic way, so that the calculations can be divided into sections, the number of which depends on the efficiency of the machine used in the execution of the algorithm. It has to be said that this modification of the basic algorithm will require the computation of the convergents as a by-product of the evaluation of the partial quotient, but there is still an overall gain in speed for the calculations, especially when many partial quotients are required, 
because most of the operations with large numbers have been eliminated. In $\S 4$ we mention another useful and important point in the modification of the basic method.

\section{A DIRECT METHOD}

Let $\alpha$ be an algebraic number with degree $k>1$, so that there is an irreducible polynomial $f(t)$ of degree $k$, with integer coefficients, such that $f(\alpha)=0 \neq f^{\prime}(\alpha)$. If $t \neq \alpha$, then, by the mean value theorem, $f(t)=$ $f(t)-f(\alpha)=(t-\alpha) f^{\prime}(\beta)$, where $\beta$ is some number lying between $\alpha$ and $t$. It follows that

$$
|t-\alpha|=\frac{|f(t)|}{\left|f^{\prime}(\beta)\right|}
$$

which is related to Newton's method for the computation of $\alpha$. The equation is also the basis of the argument used by Liouville to prove that $\alpha$ cannot be well approximated to an order which is beyond its degree $k$. For, if $t=x / y$, where $x, y$ are integers, then $y^{k} f(t)=y^{k}(x / y)$ is a nonzero integer, and so the right-hand side of (2) is at least $C / y^{k}$, where the constant $C$ can be chosen to depend only on $\alpha$, because $f^{\prime}(\beta)$ is near $f^{\prime}(\alpha) \neq 0$. Liouville's result has subsequently been extended by $A$. Thue and others, culminating in the famous Thue-Siegel-Roth theorem (see, for example, [2]).

For our purpose, we simply apply (2) with $t=x / y$ being a convergent for $\alpha$, so that, according to (1), the next complete quotient can be calculated from the function $f(t)$. More specifically, we now set $t=x_{n} / y_{n}$, the $n$th convergent to $\alpha$, so that by (1) we have

$$
\alpha^{\prime}=\alpha_{n+1}=\frac{\left|f^{\prime}(\beta)\right|}{y_{n}^{2}\left|f\left(t_{n}\right)\right|}-\frac{y_{n-1}}{y_{n}} .
$$

Here, $\beta$ is some number between $\alpha$ and $t_{n}=x_{n} / y_{n}$, and the formula is to be used for the computation of $\alpha^{\prime}$, from which new partial quotients $a_{n+1}$, $a_{n+2}, \ldots$ can be obtained. Thus, the need for new input values from an external source to calculate the complete quotient in the indirect algorithm has been eliminated by function evaluation in the direct algorithm.

We remark that $t_{n-1}$ and $t_{n}$ lie on the opposite sides of $\alpha$, so that we have bounds for $\alpha^{\prime}$ by replacing $\beta$ with $t_{n-1}$ and $t_{n}$ in (3), and the computation of such bounds will involve only rational arithmetic. When the two bounds are developed into continued fractions, all the initial partial quotients that are in agreement must also be the leading partial quotients $a_{n+1}, a_{n+2}, \ldots$ for $\alpha^{\prime}$. Thus, many new partial quotients can be obtained in one single iterative step within which function evaluations are required. The difference between the two bounds is essentially

$$
\frac{\left|f^{\prime}(\alpha)-f^{\prime}\left(t_{n}\right)\right|}{y_{n}^{2}\left|f\left(t_{n}\right)\right|} \sim \frac{\left|f^{\prime \prime}(\alpha)\right|}{y_{n}^{2}\left|f^{\prime}(\alpha)\right|},
$$

so that $\alpha^{\prime}$ can be computed to within $B / y_{n}^{2}$, where $B$ depends only on $\alpha$ and not on $n$. We may therefore compute the partial quotients $a_{n+1}, a_{n+2}, \ldots$, $a_{n+m}$ until the last convergent $x^{\prime} / y^{\prime}$ for $\alpha^{\prime}$ still satisfies $\left|x^{\prime} / y^{\prime}-\alpha^{\prime}\right|<B / y_{n}^{2}$. This shows that $y^{\prime}$ is of the order $y_{n}$, so that $y_{n+m}$ is of the order $y_{n}^{2}$. In other words, the subsequence of convergents $x / y$, obtained from iterations 
requiring function evaluation, exhibits quadratic convergence to $\alpha$. In fact, the method can be considered as a rational-arithmetic algorithm for the implementation of Newton's method for the computation of $\alpha$. In practice, we set $\beta=t_{n}$, and develop $\alpha^{\prime}$ in (3) into a continued fraction to obtain the partial quotients $a_{n+1}, a_{n+2}, \ldots, a_{n_{1}}$, which is then used to update the convergents to $x=x_{n_{1}}, y=y_{n_{1}}$, stopping for the next iteration which requires function evaluation when we reach $y_{n_{1}} \geq b y_{n}^{2}$, for some small $b=b(\alpha)>0$.

The analysis shows that the scheme works just as well for a transcendental number $\alpha$ which is given as the simple zero of a twice differentiable function $f(t)$, provided that we can calculate $f^{\prime}(t)$ and $f(t)$ to an arbitrary accuracy at rational points $t$. However, there are new considerations in the implementation of the algorithm, and we discuss these in $\S 5$. Meanwhile, we mention that it may happen that $f^{\prime}(\alpha)$ is rational, in which case there is only a problem in computing $f(t)$. For example, in our computation of the expansion for $\alpha=\pi$, we use $f(t)=\sin t$, which has the simple zero at $\alpha$, and we have $f^{\prime}(\alpha)=-1$.

\section{KHINTCHINE'S CONSTANT}

Suppose that we wish to compute the first $N$ partial quotients for a certain real number $\alpha$. As we remarked earlier, there is no a priori condition that will ensure that any precomputed value for $\alpha$ will be sufficiently accurate to deliver $a_{1}, \ldots, a_{N}$, using the basic method. This is because the sequence $\left(a_{n}\right)$ may be unbounded, so that $y_{N}>a_{1} \cdots a_{N}$ has no fixed upper bound, and hence $1 / y_{N}^{2}$ has no fixed positive lower bound for the determination of an appropriate accuracy for the input value for $\alpha$. Nevertheless, A. Khintchine [5] has proved that, for almost all $\alpha$ in the sense of Lebesgue measure, the sequence of geometric means associated with the sequence of partial quotients for $\alpha$ has a limiting value given by

$$
K=\prod_{k=1}^{\infty}\left(1+\frac{1}{k(k+2)}\right)^{\log k / \log 2} .
$$

Khintchine gave 2.6 for the value of this slowly convergent product, and D. $H$. Lehmer [10] supplied the analysis in the Euler-Maclaurin summation formula when applied to the logarithm of the product. However, Lehmer seems to have made an error in the calculation of the exponential function in the recovery of the product by giving the value 2.685550 for $K$, whereas we find that

$$
K=2.685452001065306445309714835481 \ldots \text {. }
$$

P. Lévy [11] has proved that, for almost all $\alpha$, the sequence $\left(y_{n}^{1 / n}\right)$ has the limit

$$
L=\exp \left(\pi^{2} / 12 \log 2\right)=3.275822918721811 \ldots
$$

Consequently, for almost all $\alpha$, the number of partial quotients that can be obtained by the basic method with an input approximation having accuracy of $m$ decimal digits will usually deliver $n$ partial quotients, where $n / m$ is about $\log L / 2 \log 10=\pi^{2} /(24 \log 2 \log 10)=0.97 \ldots$. In other words, we would normally require about an extra $3 \%$ in the decimal digits in the input in order to obtain the desired number of partial quotients. Nevertheless, even if $\alpha$ is not an exceptional number, we cannot claim for sure that a certain preassigned number of digits will be sufficient to deliver $N$ partial quotients. 
There is another important advantage of the direct and the indirect methods over the basic method. Suppose, for example, that we wish to compute the first 10000 partial quotients for a real number $\alpha$ using the basic method. We may wisely use 10400 decimal digits for the input value, but it is possible that even this delivers only 9990 partial quotients, and we will be forced to use a more accurate input value for $\alpha$ in order to obtain the next 10 partial quotients. However, with the new input value for the basic method, we will need to repeat all the previous calculations before we are in a position to compute the 10 new partial quotients. The only other way is to use the previously calculated partial quotients to compute also the convergents, and use the last convergent together with the new input value to update the complete quotient, but this is precisely the indirect method proposed by Lehmer. If we had used the indirect method in the first place, we could have divided the process into ' 21 sections by updating the input value with 500 new decimal digits for $\alpha$ in each section, or perhaps even 105 sections with only 100 new decimal digits in each section. The indirect method therefore allows us to have better control on the precise number of partial quotients to be calculated, as well as being a faster algorithm. Both the direct and the indirect methods can be halted and be continued again without loss. For example, we may wish to stop the calculation when enough partial quotients have been delivered, or when there is enough accuracy from the output convergents, or even when a preset computing time has been reached. Provided that we have retained the last computed partial quotient and the previous convergent, the process can always be continued again. It was our search for an algorithm which can deliver a precise number of partial quotients that led us to the direct algorithm.

\section{THE ALGORITHM}

The following is an algorithm for the direct method in the computation of the continued fraction expansion for an algebraic $\alpha$ which has been specified by the irreducible polynomial $f(t)$.

Step 1. Enter the defining polynomial $f(t)$ for the algebraic number $\alpha$.

Step 2. Use any method to find the first two convergents $t_{n}=x_{n} / y_{n}, n=0,1$, for $\alpha$.

Step 3. Enter a suitable small positive constant $b=b(\alpha)$.

Step 4. Enter a termination condition Satisfied.

Step 5. While NOT Satisfied, Do

Check that $x_{n} y_{n+1}-x_{n+1} y_{n}=(-1)^{n+1}$.

Compute the next (approximate) complete quotient, that is

$$
\alpha^{\prime}=\left|f^{\prime}\left(t_{n}\right)\right| / y_{n}^{2}\left|f\left(t_{n}\right)\right|-y_{n-1} / y_{n} .
$$

Set $B=\max \left(b y_{n}^{2}, y_{n}+1\right)$.

While $y_{n}<B$, and NOT Satisfied, Do

Replace $n \leftarrow n+1$.

Set $a_{n}=\left[\alpha^{\prime}\right]$, the next partial quotient.

Store (or collect relevant information on) $a_{n}$.

Use $a_{n}$ and the last two convergents to compute the new convergent $t_{n}=x_{n} / y_{n}$. 
Update the previous convergent $t_{n-1}=x_{n-1} / y_{n-1}$.

Replace $\alpha^{\prime} \leftarrow 1 /\left(\alpha^{\prime}-a_{n}\right)$, the next complete quotient.

Step 6. Process and print out the collected information on the expansion for $\alpha$.

Step 7. STOP

We offer the following comments on the algorithm:

- We need to have at least two convergents as initial values for the iterative scheme, and these are entered in Step 2. If $\alpha$ is initially badly approximable in the sense that its leading partial quotients all have values 1 , we may need to have more initial convergents, which will then ensure that the denominator $y_{n}$ of the convergent is not too small at the entry point for the iterations.

- In Step 3, there is some flexibility in the choice of $b=b(\alpha)$, which can be estimated from $f^{\prime}(\alpha)$ and $f^{\prime \prime}(\alpha)$, but it works quite well for many $\alpha$ by setting $b=1 / 100$. The value of $b$ is used to define the control value $B$ for the iterative process in Step 5 .

- As it has been explained, the termination condition Satisfied may be given in terms of the required number of partial quotients to be computed, or when the convergent satisfies some error bound condition, or when the number of function evaluations in the outer loop in Step 5 has been reached.

- The partial quotients $a_{n}$ are computed from the basic algorithm in the inner loop in Step 5, where they may be stored, or be discarded when relevant statistical information on them has been collected.

- As Lehmer remarked, the equation $x_{n} y_{n+1}-x_{n+1} y_{n}=(-1)^{n+1}$ provides us with "an almost infallible check", and this is being incorporated just before function evaluations.

- We set $B \geq y_{n}+1$ to ensure that at least one new partial quotient and corresponding convergent are computed in the inner loop. As to the choice of the value $b$, it is possible to set $B=y_{n}^{1.9}$ for robustness, and perhaps even some gain in speed.

- The algorithm can easily be modified so that $\alpha^{\prime}$ is evaluated to a fixed accuracy; see the following paragraph.

The algorithm for a transcendental number $\alpha$ is similar, but there are some important differences. First, $f(t)$ and $f^{\prime}(t)$ are no longer rational, so that the approximate complete quotient $\alpha^{\prime}$ cannot be computed from the algorithm using rational arithmetic. We need to specify the error within which the computation of the complete quotient $\alpha^{\prime}$ has to be maintained in each iteration. The analysis in $\S 3$ allows us to specify this error to be of the order $y_{n}^{-4}$, which will then preserve quadratic convergence. However, the notion of quadratic convergence here is being considered in terms of iterations that involves function evaluation, which will be relevant only when this evaluation is extremely difficult. In practice, it is more time-consuming in having to apply the basic continued fractions algorithm when the complete quotient is too accurately specified, and it may be better to specify the less severe bound $y_{n}^{-2(1+\delta)}$, where $0<\delta \leq 1$, and this in turn will require us to set the bound $B$ for the inner loop to be $y_{n}^{1+\delta}$. Although quadratic convergence has been sacrificed by having more function evaluations, nevertheless the arithmetic involved in the computation 
of the initial partial quotients for $\alpha^{\prime}$ will be less heavily involved, and there is usually an overall gain in speed, especially when a large number of terms in the expansion is required. Indeed, we may wish to apply the direct algorithm only as a device to remove the need for an input value, but otherwise still adopt the indirect algorithm by delivering the complete quotient each time to a certain fixed accuracy.

Finally, we mention that A. J. van der Poorten [13] has proposed a $2 \times 2$ matrix scheme in the study of continued fractions, which gives some new insight, simplification and clarification in the presentation of the theory. Some computing time can also be saved by incorporating the scheme in the implementation of the algorithms.

\section{Computation Results}

In comparison with the calculation of decimal expansions of numbers, there seem to be very few results on the calculation of continued fraction expansions. For the number $\pi$, there have been many calculations of its decimal expansion; see, for example, J. M. Borwein and P. B. Borwein [1]. On the other hand, there appear to be only seven efforts in obtaining the continued fraction expansion for $\pi=\left[a_{0}, a_{1}, \ldots\right]$, each time with an increasing number $N$ of partial quotients $a_{n}$. These are by Archimedes (240 BC) $N=1$, Tsu Ching-chih (480) $N=3, \mathrm{~J}$. Wallis [17] (1685) $N=33$, D. H. Lehmer [9, 10] $(1938,1939) \quad N=90,100$, R. S. Lehman [8] (1959) $N=1986$, and K. Y. Choong, D. E. Daykin and C. R. Rathbone [3] (1971) $N=21230$. Actually, Wallis took the calculation to $N=34$, making the mistake of giving the value 1 to $a_{34}$, the correct value for which, namely the rather large number 99 , was found by Lehmer some two-anda-half centuries later. We should also mention that $\mathbf{J}$. H. Lambert [7] proved in 1761 that $\pi$ is irrational, so that the sequence of partial quotients does not terminate, and it is of interest to recall that his proof of the irrationality of $\pi$ is based on Euler's discovery of the continued fraction expansion for

$$
e=[2,1,2,1,1,4,1,1,6,1,1,8,1, \ldots] .
$$

Lambert also checked in 1770 the calculation by Wallis up to $N=26$. In 1882 Lindemann proved that $\pi$ is transcendental, but, as far as the behavior of the sequence $\left(a_{n}\right)$ is concerned, we can only deduce from this that it is not periodic. The calculation by Lehman [8] is given in a report in the US Ballistic Research Laboratory, and, being unaware of this, G. Lochs [12] (1963) also did the calculations up to $N=968$. The calculation by Choong, Daykin and Rathbone [3] is based on the indirect method with a 25000-decimal input value.

We now give the result of our calculations of the partial quotients $a_{n}=$ $a(\alpha, n)$ of the continued fraction expansions for various numbers $\alpha$. We exclude $a_{0}=[\alpha]$ in the data, so that the partial quotients $a_{n}$ are labeled for $1 \leq n \leq N$, and we take $N=10000$. There is little point in giving the full listing of the partial quotients for any particular $\alpha$, since the sequence concerned can be obtained from the method discussed in the paper. We offer instead some statistical information in the following two tables. In Table 1 we list the number of partial quotients taking the values $a=1,2, \ldots, 10$, and also those satisfying $10<a_{n} \leq 100$ and $a_{n}>100$ in the last two columns. A. Khintchine [6] and P. Lévy [11] have proved that, for almost all $\alpha$, the set of $n$ for which 
TABLE $1 . N=10000$

\begin{tabular}{|c|c|c|c|c|c|c|c|c|c|c|c|c|}
\hline$\alpha$ & $a=1$ & $a=2$ & $a=3$ & $a=4$ & $a=5$ & $a=6$ & $a=7$ & $a=8$ & $a=9$ & $a=10$ & $10<a \leq 100$ & $a>100$ \\
\hline$\sqrt[3]{2}$ & 4173 & 1675 & 946 & 636 & 421 & 295 & 240 & 163 & 122 & 118 & 1060 & 151 \\
\hline$\sqrt[3]{2}+\sqrt{3}$ & 4129 & 1672 & 938 & 601 & 427 & 288 & 238 & 183 & 144 & 117 & 1115 & 148 \\
\hline$\pi$ & 4206 & 1672 & 882 & 597 & 443 & 282 & 224 & 186 & 143 & 123 & 1113 & 129 \\
\hline $2 \pi$ & 4133 & 1712 & 927 & 636 & 402 & 293 & 235 & 172 & 150 & 111 & 1103 & 126 \\
\hline$\pi^{2}$ & 4134 & 1706 & 948 & 581 & 401 & 302 & 232 & 185 & 138 & 117 & 1111 & 145 \\
\hline$\sqrt{\pi}$ & 4177 & 1718 & 949 & 578 & 384 & 319 & 196 & 166 & 150 & 113 & 1103 & 147 \\
\hline $\log \pi$ & 4150 & 1722 & 969 & 593 & 438 & 275 & 218 & 172 & 135 & 113 & 1093 & 122 \\
\hline$e^{\pi}$ & 4156 & 1698 & 887 & 583 & 404 & 309 & 231 & 201 & 154 & 111 & 1120 & 146 \\
\hline $\log ^{2}$ & 4149 & 1666 & 905 & 600 & 390 & 334 & 226 & 187 & 142 & 137 & 1113 & 151 \\
\hline $2^{\sqrt{2}}$ & 4192 & 1639 & 933 & 616 & 390 & 278 & 213 & 190 & 135 & 135 & 1130 & 149 \\
\hline$e \pi$ & 4165 & 1735 & 924 & 563 & 416 & 296 & 201 & 190 & 155 & 111 & 1084 & 160 \\
\hline$e+\pi$ & 4234 & 1648 & 981 & 572 & 407 & 305 & 211 & 172 & 120 & 114 & 1104 & 132 \\
\hline$\pi^{e}$ & 4116 & 1724 & 943 & 579 & 408 & 318 & 249 & 176 & 130 & 111 & 1122 & 124 \\
\hline Expectation & 4150 & 1699 & 931 & 589 & 406 & 297 & 227 & 179 & 145 & 120 & 1112 & 142 \\
\hline
\end{tabular}

$a_{n}=b$ has asymptotic density

$$
d(b)=\frac{1}{\log 2} \log \frac{(b+1)^{2}}{b(b+1)}, \quad b=1,2, \ldots .
$$

This then gives an "expectation" of the frequencies of the values taken by the partial quotients for a number $\alpha$ which is not in the exceptional set in their theorem, and we list such expectations corresponding to $N=10000$ in the last row.

If $\alpha$ is not an exceptional number for the results by Khintchine and Lévy, then, as $n \rightarrow \infty$, the two numbers $K(\alpha, n)=\left(a_{1} a_{2} \cdots a_{n}\right)^{1 / n}$ and $L(\alpha, n)=$ $\left(y_{1} y_{2} \cdots y_{n}\right)^{1 / n}$ should have the limits $K$ and $L$ given by (4) and (5). In [10] Lehmer found that $K(\pi, 100)=2.6831468 \ldots$ and $L(\pi, 100)=3.269202 \ldots$, and from these numerical results he strongly suggested that $\pi$ is not an exceptional number. Although we agree with his assessment, nevertheless we wish to point out that even if there is a convergence, the rate has to be very slow. It is easy to see that, with $n=10000$, the change in value of any single partial quotient will have an effect on the third decimal digit for the value of $K(\alpha, n)$. In fact we found that $K(\pi, 10000)$ differs from $K$ by more than $K(\pi, 100)$ does. The same remark can also be made concerning the convergence of $L(\alpha, n)$, and we only record $\left[\log _{10} y_{N}^{2}\right]$ in Table 2 , where we also include the largest partial

TABLE 2. $N=10000$

\begin{tabular}{|c|c|l|l|l|}
\hline$\alpha$ & $\max \left\{a_{n}: n \leq N\right\}$ & Missing values for $a_{n} \leq 100$ & $K(\alpha, N)$ & {$\left[2 \log _{10} y_{N}\right]$} \\
\hline$\sqrt[3]{2}$ & $a_{1990}=12737$ & $74,86,91,96,97,99,100$ & 2.65553 & 10224 \\
\hline$\sqrt[3]{2}+\sqrt{3}$ & $a_{1638}=152613$ & 70,96 & 2.71786 & 10394 \\
\hline$\pi$ & $a_{431}=20776$ & $90,91,96$ & 2.66371 & 10244 \\
\hline $2 \pi$ & $a_{425}=10387$ & 66,90 & 2.66321 & 10228 \\
\hline$\pi^{2}$ & $a_{1234}=12013$ & 91,92 & 2.69275 & 10322 \\
\hline$\sqrt{\pi}$ & $a_{8410}=121115$ & $66,76,78,81,88,93,95,97,98$ & 2.64799 & 10199 \\
\hline $\log \pi$ & $a_{3803}=12760$ & $76,79,80,82,83,87,93,98$ & 2.63385 & 10152 \\
\hline$e^{\pi}$ & $a_{8804}=150283$ & $52,66,73,80,96$ & 2.69665 & 10331 \\
\hline $\log 2$ & $a_{9168}=963664$ & $55,73,76,96,97$ & 2.72269 & 10414 \\
\hline $2^{\sqrt{2}}$ & $a_{6342}=44122$ & $79,80,81,82,91,94,97,99$ & 2.70430 & 10368 \\
\hline$e \pi$ & $a_{5409}=28656$ & $78,79,91$ & 2.67668 & 10283 \\
\hline$e+\pi$ & $a_{2720}=2978$ & $79,81,84,99$ & 2.62791 & 10148 \\
\hline$\pi^{e}$ & $a_{9014}=16323$ & $56,76,80,87,89,95,97,100$ & 2.67602 & 10271 \\
\hline
\end{tabular}


quotient found, and also the values up to 100 which are not taken by $a_{n}$ for $n \leq 10000$.

Perhaps we should not pay too much attention to the values of the largest partial quotients being computed, since it is easy to construct a number, transcendental, algebraic or rational, with any specified partial quotient at any position. Nevertheless, the following is an interesting method of finding a number with a large partial quotient. We first give an explanation for the relationship between the two large partial quotients $a(\pi, 431)=20776$ and $a(2 \pi, 425)=10387$ in Table 2. From (1) we see that if $x / y$ is a convergent to $\alpha$, then the error $|\alpha-x / y|$ is about $1 / a y^{2}$, where $a$ is the next partial quotient. It follows that if $p \mid y$ and $q \mid x$, then

$$
\left|\frac{p \alpha}{q}-\frac{x / q}{y / p}\right|=\frac{p}{q}\left|\alpha-\frac{x}{y}\right| \approx \frac{p}{q a y^{2}}=\frac{1}{(p q a)(y / p)^{2}}
$$

which indicates that the fraction $x^{\prime} / y^{\prime}$, where $x^{\prime}=x / q, y^{\prime}=y / p$, should be a convergent to $p \alpha / q$, and that the corresponding next partial quotient should have a value near pqa. In particular, if $a=a\left(\alpha, n_{0}\right)$ is already a particularly large partial quotient for $\alpha$, and $x$ and $y$ have known divisors $q$ and $p$, then we may expect $p \alpha / q$ to have an even larger partial quotient in the neighborhood of $n_{0}$. However, for the number $\pi$, the denominator $y$ of the 430th convergent $x / y$ to $\pi$ is an odd number, so that the corresponding large value of the partial quotient for $2 \pi$ is approximately halved instead. From Table 2 we also find that $a(\alpha, 1638)=152613$ when $\alpha=\sqrt[3]{2}+\sqrt{3}$. Moreover, on examining the 1637 th convergent $x / y$, we find that $y$ is divisible by 8 , and therefore $8 \alpha$ should have a partial quotient near $8 \times 152613$, and in fact $a(8 \alpha, 1670)=1220911$. Indeed, we find that 5 divides $x$, and 24 divides $y$, so that $24 \alpha / 5$ will have an even larger partial quotient, and in fact $a(24 \alpha / 5,1662)=18313683$. Our calculations here confirm what is known theoretically on the effect of multiplication of a continued fraction by a rational number; see, for example, [4, 15 and 13].

The results for the two algebraic numbers $\sqrt[3]{2}, \sqrt[3]{2}+\sqrt{3}$ were obtained by the direct method, implementing the algorithm in $\S 5$. The results for $\pi$ and $2 \pi$ were obtained by employing a modification of the algorithm, using the same transcendental function $\sin t$, but with different initial values. Similarly, the results for $\pi^{2}, \sqrt{\pi}, \log \pi, e^{\pi}$ and $\log 2$ were obtained from $\sin (\sqrt{t}), \sin \left(t^{2}\right)$, $\sin \left(e^{t}\right), \sin (\log t)$ and $e^{t}-2$, respectively. The remaining four numbers were dealt with by the indirect method.

In [10] Lehmer stated that $K(e, n) \sim C n^{1 / 3}$ as $n \rightarrow \infty$, where $C$ satisfies $3 e C^{3}=2$, and this can be proved using the explicit expansion (6). Therefore, the number $e$ lies in the exceptional set in the theorems of Khintchine and Lévy. It is also clear that quadratic irrationals are exceptional numbers, but we have not found from our computations any algebraic number with degree exceeding 2 that shows any sign of being an exceptional number, let alone one with a sequence of bounded partial quotients. D. K. L. Shiu has proposed the amusing but intractable problem of determining whether Khintchine's constant $K$ itself is not an exceptional number; in other words, whether $K(K, n) \rightarrow K$ as $n \rightarrow \infty$. All we wish to say is that the numerical value for $K$ given in (4) 
allows us to write

$$
\begin{array}{r}
K=[2,1,2,5,1,1,2,1,1,3,10,2,1,3,2,24,1,3,2,3, \\
1,1,1,90,2,1,12,1,1,1,1,5,2,6,1,6, \ldots],
\end{array}
$$

so that $K(K, 35)=3^{2 / 7} 80^{4 / 35}=2.25848 \ldots$.

\section{BIBLIOGRAPHY}

1. J. M. Borwein and P. B. Borwein, Pi and the AGM, Wiley, New York, 1987.

2. J. W. S. Cassels, An introduction to Diophantine approximations, Cambridge Tract 45 , Cambridge Univ. Press, Cambridge, 1957.

3. K. Y. Choong, D. E. Daykin, and C. R. Rathbone, Rational approximations to $\pi$, Math. Comp. 25 (1971), 387-392.

4. M. Hall, Jr., On the sum and product of continued fractions, Ann. of Math. (2) 48 (1947), 966-993.

5. A. Khintchine, Metrische Kettenbruchprobleme, Compositio Math. 1 (1935), 361-382.

6. __ Zur metrischen Kettenbruchtheorie, Compositio Math. 3 (1936), 276-285.

7. J. H. Lambert, Mémoire sur quelques propriétés remarquables des quantités transcendantes circulaires et logarithmiques, Hist. de l'Acad. Roy. de Sci. et des Belles Lettres de Berlin, Année 1761 (1768), pp. 265-322.

8. R. S. Lehman, A study of regular continued fractions, BRL Report 1066, Aberdeen Proving Ground, Maryland, February 1959.

9. D. H. Lehmer, Euclid's algorithm for large numbers, Amer. Math. Monthly 45 (1938), 227-233.

10. _ Note on an absolute constant of Khintchine, Amer. Math. Monthly 46 (1939), 148152.

11. P. Lévy, Sur le développement en fraction continue, Compositio Math. 3 (1936), 286-303.

12. G. Lochs, Die ersten 968 Kettenbruchnenner von $\pi$, Monatsh. Math. 67 (1963), 311-316.

13. A. J. van der Poorten, An introduction to continued fractions, Diophantine Analysis (J. H. Loxton and A. J. van der Poorten, eds.), Cambridge Univ. Press, Cambridge, 1986, pp. 99-138.

14. A. J. van der Poorten and J. Shallit, Folded continued fractions, J. Number Theory 40 (1992), 237-250.

15. G. N. Raney, On continued fractions and finite automata, Math. Ann. 206 (1973), 265-283.

16. H. Riesel, Prime numbers and computer methods for factorization, Birkhäuser, Boston, 1985.

17. J. Wallis, $A$ treatise of algebra, London, 1685, pp. 46-55.

Department of Mathematical SCIENCES, Loughborough University, LOUghborough, LEICESTERSHIRE LE11 3TU, UNITED KINGDOM

E-mail address: p.shiu@lut.ac.uk 